\title{
El proceso de muerte y la enfermería: un enfoque relacional. Reflexiones teóricas en torno a la atención frente a la muerte
}

\section{I ${ }^{1}$ Alba Griseida Celma Perdigon, ${ }^{2}$ Georgina Strasser |}

Resumen: En este artículo, de revisión bibliográfica y carácter téorico-conceptual, se analiza la relación existente entre las representaciones y las prácticas del profesional de enfermería en torno a la atención de pacientes en proceso de muerte. Desde un punto de vista sociológico y antropológico y de los resultados de investigaciones publicadas en el campo de la salud colectiva y de las ciencias sociales, se subraya la relación entre las prácticas del profesional de enfermería y las representaciones que de ellas se generan y la importancia de captar su lógica en el contexto social que las origina. Partiendo de reconocer que el proceso de muerte compromete asuntos biológicos, psicológicos, ideológicos, culturales, políticos e institucionales particulares, se sostiene la importancia de recuperar los componentes de los saberes que han sido invisibilizados, de identificar las repercusiones que sobre las prácticas del profesional de enfermería genera la atención del proceso de muerte de pacientes asistidos en las áreas de emergencias, ya que este proceso ha sido visto de un modo positivista, siendo analizado sólo como un hecho biológico y no como un acontecimiento social que tiene una significación cultural y que influye en las subjetividad de todos los que lo rodean.

Palabras-clave: muerte; enfermería; prácticas; representaciones sociales.

\author{
1 Fundación Gran Mariscal de \\ Ayacucho, Caracas, Venezuela. \\ Correo electrónico: albacelma@ \\ hotmail.com \\ 2 Universidad Nacional de \\ San Luis. Buenos Aires, \\ Argentina. Correo electrónico: \\ carolottoberlin@yahoo.com.ar
}

Recibido: 24/11/2014 Aprobado: 09/03/2015 


\section{Introducción}

Dice Norbert Elias que la "problemática sociológica de la muerte” aparece cuando se considera que "lo decisivo para la relación del hombre con la muerte no es sencillamente el proceso biológico en sí, sino la idea de la muerte”, idea que tiene una particularidad sociohistórica y que por lo tanto va cambiando, así como también las actitudes de los hombres frente a ésta. Este autor señala que, si bien en nuestra sociedad prima una imagen de muerte "natural", "normal", pacífica, consecuencia de la enfermedad y la vejez, en tanto que las muertes violentas (causadas por crímenes o accidentes) son vistas como casos excepcionales, incluso "en las sociedades con un nivel relativamente alto de pacificación interna, la expectativa de la muerte en la cama es más engañosa de lo que pudiera parecer a primera vista" (ELÍAS, 2011, p. 85). La muerte es un hecho social paulatinamente institucionalizado al trasladarse del hogar a los hospitales, sea por aliviar el sufrimiento del que agoniza, o por miedo de morir sin atención; el número de pacientes que muere en las instituciones sanitarias es una tendencia que va en aumento, "la muerte, que en un $80 \%$ de los casos sobreviene a partir de ahora en el hospital o en la clínica, está totalmente medicalizada" (PROST; VINCENT, 1991, p. 138). Por institucionalizado se hace referencia a una instauración social que tiene por objetivo establecer categorías de conductas, actuaciones, relaciones y vínculos que se dan entre los individuos que la forman, a modo de acciones técnicas, y las funciones normadas por el hospital o del ejercicio de la profesión, como los protocolos de actuación.

La evolución de las ciencias y los saberes técnicos expresa la dominación del ser humano sobre la biología intentando alargar la vida hasta sus últimas circunstancias, donde la "incapacidad para suprimir la muerte es vivida como un fracaso de su saber y de su poder" (PROST; VINCENT, 1991, p. 347). Philippe Ariés remarca que ahora lo cotidiano es morir en el hospital, rodeado no tanto de seres queridos como de un equipo de especialistas en morir (ARIÉS, 1984), siendo el profesional de enfermería quien en su labor hace frente a la atención de estos pacientes que agonizan.

Para el profesional de enfermería toda práctica está orientada y emerge de representaciones imposibles de desligar de su labor diaria; sin embargo, la mayoría de sus acciones son vistas como técnicas o científicas, sin darle el valor social que ellas connotan. Los profesionales de la salud, durante su desempeño laboral, 
fusionan sus saberes técnicos y científicos con el conocimiento empírico y social,

pero, como generalmente se atribuye a las prácticas una racionalidad técnica, lo que se documenta en las historias clínicas de los pacientes y en los reportes de la guardia de trabajo en las áreas clínicas son las técnicas y procedimientos y no los saberes prácticos utilizados para afrontar los acontecimientos en las emergencias. Por muy objetivas que sean las técnicas, son realizadas por seres sociales que interactúan en un colectivo, de allí la importancia de un enfoque relacional para abordar los saberes y las prácticas de los profesionales de enfermería.

Este texto forma parte del marco teórico de un proyecto de tesis doctoral, que se propone abordar desde un enfoque cualitativo las repercusiones que la atención de pacientes en proceso de muerte tiene en las representaciones y las prácticas de los profesionales de enfermería que trabajan en los servicios de emergencia de un hospital.

Elegimos como conceptos centrales el de "representaciones sociales" de Serge Moscovici y el de habitus planteado por Pierre Bourdieu, junto al enfoque relacional referenciado por Eduardo Menéndez. Se utiliza bibliografía de diversas disciplinas como la antropología, sociología y ciencias de la salud, articulando así un conjunto de conceptos y conocimientos como fuente valiosa para la reflexión crítica en este campo. Se realizó una revisión de investigaciones relacionadas con el tema, publicadas en libros, revistas y artículos científicos del área de la salud colectiva y de las ciencias sociales.

\section{La muerte y la enfermería}

La muerte es un acontecimiento inevitable y universal, pero "las actitudes hacia los moribundos y hacia la muerte [...] no son ni inalterables ni accidentales", son peculiaridades de sociedades determinadas (ELÍAS, 2011, p. 131). Los padecimientos, los modos de enfermar y de morir son procesos históricos y socialmente construidos según las condiciones de vida de los conjuntos sociales y sus modos de afrontarlos, produciendo sentidos y significaciones individuales y colectivas que se exteriorizan de diferentes formas (GRIMBERG, 1998, MENÉNDEZ, 1990).

La muerte es un hecho social que ha pasado a ser institucionalizado: La instauración de ella en los hospitales, ya sea por deseo de prolongar la vida o por el miedo de morir sin atención, ha alejado este acontecimiento del entorno 
colectivo donde era visto como un evento natural: "Y entre tanta tecnología y sin alma, entre tantos objetos inanimados y casi ningún sujeto ¿cómo percibir el rasgo de humanidad en todo acto médico?” (GHERARDI, 2007, p. 29). Tanta tecnología y omnipotencia de la ciencia oculta el padecimiento humano no solo del enfermo sino del que lo atiende.

Al respecto, Eduardo Menéndez señala que en los últimos tiempos "desaparecen los principales rituales de mortalidad, como parte del proceso de ocultamiento y negación de la muerte que caracterizaría a la sociedad occidental" (MENÉNDEZ, 2006, p. 152). La muerte ha pasado de ser un acontecimiento natural y social, a ser asumido como algo vergonzoso e individual, que quiere ser evitado (GRIMBERG, 1992). La muerte se ha convertido en un asunto traumático: "los grupos sociales actuales no sólo han desarrollados rituales de evitación y ocultamiento respecto a la muerte, sino que desarrollaron rituales para reducir la muerte a enfermedad" (MENÉNDEZ, 2006, p. 153). El profesional de enfermería no queda al margen de estos rituales de evitación y reducción de la muerte a enfermedad, percibiendo el fallecimiento del paciente como un fracaso de sus acciones y esfuerzos terapéuticos por salvar una vida (CHACÓN; GRAU, 1997; FRUTOS et al., 2007; MAZA et al., 2009).

En numerosos trabajos que tratan el tema de la muerte en el ámbito de la salud, como los realizados por Castanedo (2010), Codorniu (2011), Iglesias (2010) y Vargas (2011) se explica cómo se debe tratar al paciente, los protocolos de actuación, las acciones apropiadas en la atención, y hasta resaltan la necesidad de formación del profesional de enfermería en los cuidados hacia el final de la vida y la muerte. Raras veces se habla del inevitable fin de la vida o de lo que debe hacer la enfermera para ayudar a una persona a reducir sus molestias y afrontar la muerte con coraje o dignidad (HENDERSON, 1995), pero más raro es encontrar análisis que hablen sobré qué le ocurre al personal de enfermería al enfrentar estos procesos. La confrontación de la muerte por parte del personal de enfermería, está impregnada de sentimientos tales como impotencia, angustia, sufrimiento, miedo, que interfirieron en la asistencia prestada al enfermo y su familia (SOUZA; SOUZA et al., 2013); "tanto es así, que los estudiantes de enfermería describen estas experiencias [la muerte] como 'lo malo' de la profesión (KIGER, 1994). Los temas más recurrentes en los diarios de prácticas que realizan los alumnos son principalmente la muerte súbita y la vulnerabilidad ante 
la misma, concediendo gran importancia a la necesidad de apoyo psicológico ante el impacto de estas vivencias" (BENBUNAN et al., 2007, p. 199).

El personal de enfermería es un sujeto que siente y padece, atravesado por representaciones sociales y por sus prácticas (porque como señala Menéndez (1975), los saberes no pueden ser negados ni separados de las prácticas). El “decidir si se deja morir o se intenta que viva se basa en la experiencia del clínico, en sus creencias, en consideraciones sociales 'porque es joven y tiene dos hijos pequeños', en la percepción de su red social” (COMELLES; PERDIGUERO, 2000, p. 315).

\section{Repensando la labor de enfermería desde los conceptos de representaciones sociales y habitus o prácticas}

Por representaciones sociales se entiende las (re-)interpretaciones y codificaciones de la realidad donde se articulan lo psicológico y lo social y que orientan las prácticas que realiza el profesional de enfermería en su quehacer cotidiano, el cual no puede ser tomado como una práctica técnica o un saber netamente científico, sino que va más allá de sus funciones aprendidas en las escuelas de enfermería incorporando las experiencias de su práctica laboral. Además, estas representaciones se conforman a partir de sus otros saberes culturales, sociales, creencias y experiencias pasadas. Las representaciones orientan consciente o inconscientemente sus labores de atención a pacientes en proceso de muerte.

Tomando las ideas de Durkheim formuladas a finales del siglo XIX, las representaciones se desglosan de las relaciones que se establecen entre los sujetos o grupos sociales (2000). En los servicios de emergencias hospitalarios se relacionan distintos grupos de individuos, como el de los profesionales y los pacientes y sus familiares, conformándose a partir de esta interacción el entorno de las prácticas profesionales cotidianas para dar respuestas a las necesidades de atención de los pacientes. Expresado de modo claro por Grimberg, "los procesos de salud enfermedad no solo emergen de esas condiciones-socio-económicaspolíticas-, sino que son objeto de la construcción de los saberes y prácticas de los conjuntos sociales incluidos los especialistas (curadores-funcionarios de salud), que posibilitan su definición, su reconocimiento así como la forma organizada de atención" (GRIMBERG, 1992, p. 4).

Las prácticas que realiza el profesional de enfermería al atender a los pacientes en proceso de muerte en los servicios de emergencia se sitúan "sobre el cruce de 
las coacciones sociales que pasan sobre el individuo y de los deseos o carencias que hacen eco de ellas. Las representaciones expresan y permiten trascender sus contradicciones" (JODELET, 1986, p. 479). Contradicciones que se dan en la toma de decisiones al momento de la atención del paciente y que se manifiestan en sus representaciones y prácticas. "Las prácticas no se construyen solo desde afuera y por la fuerza, sino desde adentro de los conjuntos a través de procesos conscientes y no conscientes" (GRIMBERG, 1992, p. 6), otorgándole a estas el componente de subjetividad, que las convierte más que en un acto técnico en un acto social.

Las representaciones pueden generar cambios en las prácticas y llegar a hacerse tan cotidianas que se invisibilizan, lo que afecta la actuación de los individuos y los grupos que se encuentran inmersos en esa relación profesional/paciente. "Las enfermedades producen sentidos y significaciones subjetivas y colectivas que operan de diversas maneras en los procesos salud/enfermedad" (NAVARRO, 1983, p. 103). Retomando las palabras de Menéndez, "las representaciones establecen la guía para la acción, la cual se resuelve en la actuación. La práctica puede modificarse situacionalmente frente a las variaciones que presentan los diferentes cuadros de una enfermedad y frente a los pacientes tomados como individuos en función al tipo de institución en la que trabaje el médico" (MENÉNDEZ, 1996, p. 55).

Las prácticas no pueden ser pensadas separadas del entorno que las origina ya que esta forma parte del accionar de dichas prácticas. La necesidad de atención y las condiciones del paciente en los servicios de emergencia determinan un tipo de trabajo técnico y científico que surge subordinado por la acción rápida y urgente, lo que obliga a dar respuestas que solucionen las situaciones, emergiendo prácticas impregnadas de representaciones donde se cruzan los saberes técnicos y los saberes sociales. Uno de los aspectos centrales para abordar este tema es el de comprender que la visión simple de la realidad no es capaz de descifrar, por sí misma, los principios de la lógica práctica: "lo social está conformado por relaciones objetivas, pero que también los individuos tienen un conocimiento práctico de esas relaciones -una manera de percibirlas, de evaluarlas, de sentirlas de vivirlas-, e invierten ese conocimiento práctico en sus actividades ordinarias" (GUTIÉRREZ, 2000, p. 12). Es así que las prácticas observadas en el profesional de enfermería al momento de atender a un paciente en proceso de muerte, siempre van a estar ligadas no sólo al contexto y las circunstancias en los que 
acontecimientos se desarrollan, sino que van a estar afectadas igualmente por

factores externos a ella como pueden ser las normativas institucionales, creencias religiosas o su entorno social.

Los saberes y las prácticas en enfermería son estructurados en un nivel científico y técnico y sus funciones son medidas por la eficacia en la curación del paciente y su recuperación. El profesional de enfermería se autopercibe como integrante de la labor de cuidar, "mientras que la profesión ha luchado para ser más científica y más profesional” (DAVIS, 2006, p. 2). Lo delicado y específico de sus labores exige que su saber requiera una preparación más compleja y superior a la que normalmente estaría sometido cualquier ejecutor de tareas ya preestablecidas (ASSUNÇÃO et al., 2011).

La enfermería como rama de la salud, se dedica al cuidado integral del ser humano y basa sus fundamentos en el saber de las Ciencias Médicas. El Consejo Internacional de Enfermeras (CIE) señala que la enfermería es una profesión que

[...] abarca los cuidados, autónomos y en colaboración, que se prestan a las personas de todas las edades, familias, grupos y comunidades, enfermos o sanos, en todos los contextos, e incluye la promoción de la salud, la prevención de la enfermedad, y los cuidados de los enfermos, discapacitados, y personas moribundas (CIE, 2014).

Ese cuidado prestado por el personal de enfermería y la forma en que es organizado constituye en sí una disciplina sanitaria y humanística, que implementa en su práctica el saber de las ciencias físicas, biológicas, sociales, entre muchas otras. El propio saber del personal de enfermería está conformado como una fusión de conocimientos disciplinarios y sistemáticos, sobre una materia concreta de la ciencia que es el cuidado (GRACIA; MARTINEZ, 2001).

El carácter indiscutible otorgado al conocimiento técnico y científico en el área de la salud (hegemonía médica) puede ser un obstáculo en la posibilidad de cuestionamiento de algunas de sus prácticas, a pesar de que uno de los elementos inherentes del conocimiento científico es, precisamente, la revisión permanente de procedimientos y resultados. También es cierto, que ciertas acciones del profesional de enfermería no necesariamente tienen que ver con la ortodoxia de la disciplina y que estas prácticas no convencionales pueden verse amparadas en el prestigio científico.

Pero todo en sus prácticas no debe ser visto solo como un trabajo terapéutico. No es posible desligar las representaciones de la labor diaria de estos profesionales a pesar de que muchas de sus acciones sean definidas como institucionales, sin 
darle el valor social que ellas connotan. Al respecto, Menéndez refiere que "en todos los contextos las instituciones asistenciales, de protección o de control, estructuran una racionalidad que no es exclusivamente técnica ni científica, sino también sociocultural” (MENÉNDEZ, 1994, p. 73). En palabras de Bourdieu, y citando su concepto de habitus, se puede decir que "los esquemas de percepción, de pensamiento y de acción, tienden con más seguridad que todas las reglas formales y todas las normas explicitas, a garantizar la conformidad de la prácticas y su constancia a través del tiempo" (BOURDIEU, 2007, p. 89). Así podemos entender que no toda labor que realice el profesional de enfermería en su ejercicio profesional es producto de su saber técnico o de su formación académica, sino que en sus prácticas se expresa el habitus, rector de su accionar como una composición de operaciones producto de su subjetividad pero también de los saberes formales o institucionales. En el concepto de habitus hay una ontología de las destrezas y capacidades adquiridas, resultado de una racionalidad práctica que no puede ser exclusivamente pensada como producto de la razón técnica, científica o técnica (CHAUVIRE; FONTAINE, 2008). Esto nos sirve para entender cómo las prácticas ejecutadas por el profesional de enfermería al momento de atender pacientes en proceso de muerte están marcadas por un sesgo de sentidos definidos no solamente por los saberes técnicos sino que a su vez se reflejan en ellas los esquemas de percepciones, de apreciación y de acción interiorizados y que predisponen su actuar y accionar frente al paciente que agoniza. Los habitus funcionan como

[...] principios generadores de prácticas y de representaciones que pueden ser objetivamente adaptadas a sus objetivos sin suponer el punto de mira consciente de fines y el dominio expreso de las operaciones necesarias para alcanzarlas, objetivamente "reguladas" y regulares sin ser para nada el producto de la obediencia a reglas, y, siendo todo eso colectivamente orquestadas sin ser el producto de la acción organizadora de un director de orquesta (BOURDIEU, 2007, p. 86).

Los habitus nos hacen ver como evidentes o naturales rasgos de las prácticas del profesional de enfermería que son orientadas por las representaciones que los mismos profesionales han creado de su entorno y que luego quedan invisibilizados o son naturalizados en sus labores. Son posibilidades objetivas, propensas a renovarse y a influir en las prácticas y las representaciones que moldean de manera duradera. El habitus es originado por el condicionamiento social e histórico y a su vez es creador de prácticas, adaptadas al raciocinio del campo social implicado, por lo tanto permitidas y delimitadas por ellas (CHAUVIRE; FONTAINE, 2008). 
Al momento de su desempeño laboral tanto el profesional de enfermería como otros profesionales de la salud entrama sus saberes técnicos y científicos con el conocimiento empírico y social. Una de las manifestaciones en las que se observan prácticas que van más allá del protocolo normativo institucional, es la costumbre que se da en los centros hospitalarios en Venezuela, experiencia vivenciada directamente por la autora de este trabajo, es que ningún paciente es sacado de la sala de operaciones con los pies en dirección hacia la puerta, ya que se considera que de ese modo solo salen los cadáveres. Es una práctica que se basa en el acervo cultural y que evidentemente no contiene ningún motivo científico racional que lo justifique. El cuidado que ofrece el profesional de enfermería a un paciente moribundo y agónico rompe, en algunas ocasiones, las barreras de las normas. Envueltos en los saberes sociales y culturales arraigados, este personal intenta ceñirse a un protocolo de actuación que institucionaliza sus prácticas, mientras que se proporciona una atención que va más allá de fundamentos biológicos y de la racionalidad técnica o científica.

Cuando un paciente agoniza hace aflorar en otros un sentimiento de angustia, miedo o hasta resignación que conllevan a recordar lo frágil que puede ser la vida. Es en esos momentos cuando el profesional de enfermería reacciona más allá de los conocimientos técnicos y sale a relucir su dimensión social y cultural. Esta dimensión se manifiesta, por ejemplo, dando consuelo religioso, psicológico y su saber se convierte en humano y el dolor ajeno se convierte en propio. La muerte también lleva a generar aislamiento y desapego en algunos profesionales (VARGAS et al., 2011), y estos sentimientos son utilizados como estrategias para evitar afrontar el dolor de la pérdida, a pesar de ser ajena. "La muerte, como fenómeno inevitable, constituye un poderoso estímulo ansiogénico capaz de afectar a actitudes y comportamientos e incidir en la calidad de los cuidados del profesional de enfermería" (SÁBADO; GUIX, 2001, p. 20). Lidiar con las limitaciones que impone la muerte, el sufrimiento y el dolor de un semejante es una situación difícil y desgastante física y psicológicamente (ASSUNÇÃO et al., 2011).

De acuerdo al enfoque relacional para abordar los saberes y las prácticas en los cuales se entrecruza lo biológico con lo social, Menéndez indica que

[...] enfermar, morir, atender la enfermedad y la muerte deben ser pensados como procesos que no sólo se definen a partir de profesiones e instituciones dadas, específicas y especializadas, sino como hechos sociales respecto a los cuales los conjuntos sociales necesitan construir acciones, técnicas e ideologías, una parte de las cuales se organizan profesionalmente (MENÉNDEZ, 1994, p. 71). 
Los saberes de los enfermeros en tanto son significados socialmente construidos, están insertos en un contexto histórico que los condiciona y les otorga características de sentido en el entorno donde estos saberes son manifestados en forma de prácticas. Como lo expresa Grimberg, "decir que su carácter es social significa plantear estos procesos en relación a la totalidad de la vida social y sus determinaciones" (GRIMBERG, 1992, p. 5).

$\mathrm{Al}$ atender en los servicios de salud a los pacientes que se encuentran en proceso de muerte los saberes técnicos empleados para intentar salvar la vida se conjugan con el entorno, el momento, las circunstancias y las acciones en esta lucha de la vida y la muerte entre sujetos y grupo de trabajo. Estas situaciones se transforman de mero acto técnico a ser un acto de alto contenido social: "Las relaciones de los individuos y de los grupos sociales con su cuerpo, su sufrir, su padecer, su muerte, y con el cuerpo, el sufrimiento, el padecimiento y la muerte de los otros son relaciones sociales" (LUZ, 1997, p. 13). El trabajo del profesional de enfermería va más allá de la sola repetición de procedimientos. La interacción entre el profesional y los pacientes va modificando las tareas, las rutinas y hasta los protocolos de acción. Los trabajadores usan los saberes construidos desde la experiencia y las representaciones sociales para afrontar situaciones difíciles que ya sea por las mismas condiciones institucionales o por las características de la situación no pueden ser resueltas con solo el saber científico y la tecnología (OSORIO et al., 2011).

$\mathrm{Al}$ estar frente a un paciente que agoniza, el profesional de enfermería inicia maniobras científicas y tecnológicas integradas a un grupo institucionalizado que realiza sus funciones en pro de salvar la vida del paciente, "mas la tendencia a homogenización técnica no elimina la presencia de los procesos ideológicosculturales" (MENÉNDEZ, 1996, p. 17), y son estos procesos ideológicos y culturales los que conllevan cambios en las prácticas de estos profesionales.

Visto desde el punto de vista formativo, el saber científico anula el saber socio cultural. Lo que se documenta en las áreas clínicas, como en las historias del paciente, en los textos de formación académicas, en artículos científicos, entre otros, son las técnicas y procedimientos y no los saberes prácticos utilizados para afrontar hechos biológicos como la muerte de otro ser humano. Por ello, resaltamos la importancia del enfoque relacional de los saberes y las prácticas en el profesional de enfermería, ya que por muy objetivas que sean las técnicas, 
son realizadas por seres sociales que interactúan en un colectivo. "La sociedad

modela al individuo, lo influye, a menudo también lo ataca, pero también lo define" (DI TELLA, 2007, p. 13).

\section{Conclusión}

Desde un abordaje teórico-conceptual, este artículo desarrolla la idea de que el trabajo del profesional de enfermería se lleva a cabo en un contexto de relaciones que influyen en sus representaciones y prácticas profesionales al atender a pacientes en proceso de muerte en los servicios de emergencia. El enfrentar la muerte es un proceso difícil y la atención a los seres humanos es compleja y llena de connotaciones bio-psico-sociales que deben ser tenidas en cuenta tanto en la formación del personal de enfermería como en la organización de las prácticas de atención. Teniendo en cuenta la tendencia en continuo aumento de la atención del proceso de muerte en los hospitales, y que esta institucionalización implica una racionalización técnica y una reglamentación en protocolos de acción de las conductas y relaciones entre los sujetos involucrados, queda relegado a un segundo plano el componente de subjetividad de las prácticas profesionales, que hace de ellas un acto social, más que en un acto técnico.

A partir de la revisión de investigaciones publicadas en el campo de la salud colectiva y de las ciencias sociales, se destaca que, dado que el personal de enfermería interactúa con el paciente y muchas otras personas en el proceso de brindar cuidados de enfermería adecuados, inevitablemente su cultura, sus actitudes y creencias repercutirán en sus decisiones y prácticas. Este modo de ver los saberes del profesional nos debe permitir salir del argumento positivista que considera las prácticas para el cuidado de la salud y la atención del proceso de muerte regidas sólo por una racionalidad biológica y técnica, y que deja de percibir los condicionamientos sociales de tales prácticas al momento de atender el proceso de muerte de los pacientes.

Se considera entonces la importancia de realizar investigaciones sobre la atención de los procesos de muerte mediante una metodología cualitativa, que propicie identificar y describir las múltiples relaciones entre los sentidos, las emociones, los saberes y acciones del personal de enfermería, intentando vincular sus prácticas con sus vivencias y representaciones socialmente construidas. ${ }^{1}$ 


\section{Referencias}

ARIÉS, P. El hombre ante la muerte. Madrid: Taurus, 1984.

ASSUNÇÃO, A. A. Trabalhar na Saúde. Experiencias cotidianas e desafíos para a gestao do trabalho e do emprego. Rio de Janeiro: Fiocruz, 2011.

BENBUNAN, B. et al. Afrontamiento del dolor y la muerte en estudiantes de enfermería: una propuesta de intervención. International Journal of clinical and Health Psychology, v.7, n.1, p. 197-205, 2007.

BOURDIEU, P.; PASSERON, J.; CHAMBOREDON, J. El oficio del Sociólogo. Madrid: Siglo XXI, 1975.

BOURDIEU, P. El sentido práctico de las cosas. Buenos Aires: Siglo XXI, 2007.

CASTANEDO, I.; SUÁREZ, C.; VIVAR, C. Capacitación y afrontamiento de las enfermeras de urgencias ante la muerte y el duelo. Enfermería Docente, España, n. 91, p. 16-18, 2010. Disponible en: <www.index-f.com/edocente/91pdf/91-016.pdf> Acceso en: 7 Mar. 2013.

CARMONA, Z.; BRACHO, C. La muerte y el duelo en el equipo de salud. Revista de Salud Pública, [online] v. 2, n. 2, p.14-23, 2008. Disponible en: <www.saludpublica.fcm.unc.edu. $\mathrm{ar} /$ sites/default/.../art2_carmona> Acceso en: 7 Mar 2013.

CHACÓN, M.; GRAU, J. Burnout en enfermeros que brindan atención a pacientes oncológicos. Rev Cubana Oncol, [online] v. 13, n. 2, p. 118-125, 1997 Disponible en: <http://bvs.sld.cu/revistas/onc/vol13_2_97/onc10297.htm> Acceso en: 19 Dic. 2013.

CHAUVIRÉ, C.; FONTAINE, O. El Vocabulario de Bourdieu. Buenos Aires: Atuel, 2008. COMELLES, J.; PERDIGUERO, E. Medicina y cultura: estudios entre la antropología y la medicina. Barcelona: Bellaterra, 2000.

CONSEJO INTERNACIONAL DE ENFERMERAS. La definición de enfermería. CIE; 2014 Disponible en: <http://www.icn.ch/es/about-icn/icn-definition-of-nursing > Acceso en: 31 oct 2014 .

DAVIS, A. El cuidar y la ética del cuidar en el siglo XXI: qué sabemos y que debemos cuestionar. Revista del Col-legi oficial d'infermeres de Barcelona [online] S/N, p. 1-25, 2006 Disponible en: <http://www.coib.org/uploadsBO/noticia/documents/ANNE\%20 DAVIS\%20CASTELL\%C3\%A0_DEF> Acceso en: 20 Dic 2012.

DI TELlA, T. Conceptos Sociológicos Básicos. In: DI TELLA, T.; LUCCHINI, C. (comp.). Nociones básicas de Sociología. 4a Ed. Buenos Aires: Biblos, 2007.

DURKHEIM, E. Sociología y Filosofía. Madrid: Mino y Dávila, 2000.

ELÍAS, N. La soledad de los moribundos. México: Fondo de Cultura Económica, 2011. 
FRUTOS, M. et al. La persona en proceso de muerte. Revista Enfermería Global [online] v. 6, n. 1, p. 1-14, 2007. Disponible en: <http://revistas.um.es/eglobal/article/view/208> Acceso en: 12 Oct 2012.

GHERARDI, C. Vida y Muerte en terapia intensiva: estrategias para conocer y participar de las decisiones. Buenos Aires: Biblos, 2007.

GARCÍA MARTÍN-CARO, C.; MARTÍNEZ, N. Historia de la enfermería: evolución histórica del cuidado enfermero. Madrid: Harcourt, 2001.

GUTIÉRREZ, A. Las prácticas sociales: una introducción a Pierre Bourdieu. Buenos Aires: Eduvim, 2000.

GRIMBERG, M. Proceso salud-enfermedad-atención y hegemonia. Guía para la discusión. Doc. de trabajo. Buenos Aires: ICA-UBA, 1992.

GRIMBERG, M. VIH / Sida y proceso salud-enfermedad-atención: construcción social y relaciones de hegemonía. In: SEMINARIO TALLER DE CAPACITACIÓN DE FORMADORES. Buenos Aires: Lusida, 1992. p. 24-31.

HENDERSON, V. La naturaleza de la enfermería: reflexiones 25 años después. Madrid: Mc Graw Hill Interamericana. 1995.

JODELET, D. La representación social: fenómeno, concepto y teoría. In: MOSCOVICI, S. (comp.). Psicología Social II. Barcelona: Paidós, 1986.

LUZ, M. Natural, Racional, Social. Buenos Aires: Lugar, 1997.

MAZA, M.; ZAVALA, M.; MERINO, J. Actitud del profesional de enfermería ante la muerte de pacientes. Cienc. enferm. [online] v. 15, n. 1, p. 39-48, 2009. Disponible en: <http:// www.scielo.cl/scielo.php?script=sci_arttext \&pid $=$ S0717-95532009000100006\&lng $=$ es. Acceso en: 07 Feb. 2013.

MENÉNDEZ, E. Morir de Alcohol. Saber y hegemonía medica. México: Alianza Editorial Mexicana, 1990.

MENÉNDEZ, E. La enfermedad y la curación ¿qué es medicina tradicional? Alteridades, Universidad Autónoma Metropolitana Unidad Iztapalapa México, v. 4, n. 7, p. 71-83, 1994. Disponible en: <http://biblioteca.ues.edu.sv/revistas/10800276-8.pdf> Acceso en: 26 Nov. 2012.

MENÉNDEZ, E.; DI PARDO, R. De algunos alcoholismos y algunos saberes: atención primaria y proceso de alcoholización. México: CIESAS, 1996.

MENÉNDEZ, E. Desaparición, resignificación o nuevos desarrollos de lazos y rituales sociales. Relaciones, v. 27, n. 107, p. 147-178, 2006.

OSORIO, C. et al. O trabalho cotidiano em hospitais: $O$ ponto de vista da atividade de enfermagem. In: ASSUNÇÃO, A. A.; BRITO, J. (Org.) Trabalhar na Saúde: experiencias cotidianas e desafíos para a gestao do trabalho e do emprego. Rio de Janeiro: Fiocruz, 2011. 
PROST, A.; VINCENT, G. La vida privada en el siglo XX. In: ARIÈS, P.; DUBY, G. Historia de la vida privada. Madrid, Altea, Taurus, Alfaguara, 1991. Tomo 9.

SÁBADO, T.; GUIX, E. Ansiedad ante la muerte: efectos de un curso de formación en enfermeras y auxiliares de enfermería. Enferm. Clin. [online] v. 11, n. 3, p. 104-109, 2001. Disponible en: <http://www.elsevier.es/es/revistas/enfermeria-clinica-35/ansiedad-muerteefectos-uncuR. S.o-formacion-enfermeras-13016475-originales-2001? bd=1>

SCHWARTZ, H.; JACOBS, J. Sociología cualitativa: método para la reconstrucción de la realidad. México: Trillas, 1984.

SOUZA E SOUZA, L. P. et al. La muerte y el proceso de morir: sentimientos manifestados por los enfermeros. Enferm. glob., v. 12, p. 222-229, 2013.

VARGAS, D. et al. Percepción de enfermería en torno a la muerte de pacientes en etapa terminal. Revista de Enfermería del Instituto Mexicano del seguro Social, [online] v. 16, n. 1, p. 37-44, 2011 Disponible en: <http://revistaenfermeria.imss.gob.mx/index. php? option $=$ com_multicategories $\& v$ view $=$ article $\& i d=150:$ percepcion-de-enfermeriaen-torno-a-la-muerte-de-pacientes-en-etapa-terminal \&catid = 108: experiencias-de-la-practica\&Itemid= 80> Acceso en: 07 Dic 2012.

\section{Nota}

${ }^{1}$ A. G. C. Perdigon: planteamiento del tema, revisión bibliográfica, análisis y escritura del manuscrito. G. Strasser: corrección del análisis teórico y de la redacción; corrección del formato conforme a la normativa de la revista. 
O processo de morte e a enfermagem: uma abordagem relacional. Reflexóes teóricas sobre os cuidados antes da morte

Neste artigo, de revisão de literatura e natureza teóricoconceitual, analisa-se a relação entre representações e práticas dos profissionais de enfermagem quanto aos cuidados do paciente no processo de morte. Do ponto de vista sociológico e antropológico, e com base nos resultados da pesquisa publicada no campo da saúde coletiva e ciências sociais, destaca-se a relação entre as práticas do enfermeiro e as representações geradas, salientando a importância de compreender sua lógica no contexto social que as origina. Começando a reconhecer que o processo de morte envolve aspectos biológicos, psicológicos, ideológicos, culturais, políticos e institucionais, salienta-se a importância de recuperar os componentes do conhecimento que foram invisibilizados, de identificar as repercussões que na prática do profissional de enfermagem gera a atenção do processo de morte de pacientes atendidos nas áreas de emergência, já que esse processo tem sido visto de maneira positivista, sendo analisado apenas como um fato biológico e não como um acontecimento social que tem um significado cultural e influencia na subjetividade de todos ao redor.

> Palavras-chave: morte; enfermagem; práticas; representações sociais. 


\section{Dying process and nursing: a relational approach. Theoretical reflections on assistance dealing with death}

In this literature review article of theoretical-conceptual nature, the relationship between representations and practices of nursing professionals regarding patient care in the process of death is analyzed. From a sociological and anthropological point of view and based on the results of published research in the field of collective health and social sciences, the relationship between nursing practices and the representations generated stresses the importance to grasp its logic in the social context it gives rise.

Starting to recognize that the process of death committed biological, psychological, ideological, cultural, political and institutional particular issues, the importance of recovering the components of knowledge that have been invisible, to identify the impact argues that on professional practices Nursing generates the attention of the death process of patients assisted in the areas of emergency, as this process has been seen in a positivist way, being analyzed only as a biological fact and not as a social event has cultural significance and influences in the subjectivity of everyone around.

> Key words: death; nursing; practices; social representations. 